\title{
THE POINCARÉ CONJECTURE
}

\section{A PROBLEM SOLVED AFTER A CENTURY OF NEW IDEAS AND CONTINUED WORK}

\author{
MARÍA TERESA LOZANO IMÍZCOZ
}

The Poincaré conjecture is a topological problem established in 1904 by the French mathematician Henri Poincaré. It characterises three-dimensional spheres in a very simple way. It uses only the first invariant of algebraic topology - the fundamental group - which was also defined and studied by Poincaré. The conjecture implies that if a space does not have essential holes, then it is a sphere. This problem was directly solved between 2002 and 2003 by Grigori Perelman, and as a consequence of his demonstration of the Thurston geometrisation conjecture, which culminated in the path proposed by Richard Hamilton.

Keywords: topology, sphere, fundamental group, Riemannian geometry, Ricci flow.

\section{RECOGNIZED WORK}

The Poincare conjecture is the only millennium problem that has been solved so far. On 18 March 2010, the Clay Mathematics Institute announced a prize of one million dollars for the Russian mathematician Grigori Perelman. Four years before, Perelman had been awarded a Fields Medal at the International Congress of Mathematicians held in Madrid in 2006, for his contributions to geometry and his revolutionary vision of the analytical and geometric structure of the Ricci flow, but he did not accept his Fields Medal or his millennium prize.

Perelman announced in three preprints, published between 2002 and 2003 in the open access database arXiv, the solution to the Thurston geometrisation conjecture, which contains the Poincaré conjecture as a particular case. The two first texts, together with his unpublished work as well as the work, published after 2003, of other mathematicians, proved the conjecture of geometrisation. The third text, which assumes the essential results of the other two, offers a direct verification of the Poincaré conjecture.

Perelman's solution is based on ideas of Richard Hamilton and uses the Ricci flow, which employs differential equations to connect curvatures with the metric's variation. In fact, Hamilton received the prestigious Clay Research Award in 2003 for the discovery and development of the Ricci flow, one of the most powerful tools in geometric analysis.

\section{INITIAL FRAMEWORK OF THE CONJECTURE: TOPOLOGY}

The Poincaré conjecture is framed within one of the most abstract branches of mathematics, topology. Its creator, the French mathematician Jules Henri Poincaré, called it «analysis situs»; and it is the field of mathematics that characterises some of the qualitative properties of objects, those that remain after continuous deformation, that is, those that are smooth, without breaking points, cuts, or identifications. It is a type of soft geometry, rather than a rigid one. This original idea was the fruit of a mind with an extraordinary ability for spatial abstraction.

We can say that in topology two objects -topological spaces - are equivalent or homeomorphic when one can be obtained from the other through a continuous deformation, that is, when there is a biunivocal and bicontinuous correspondence between 


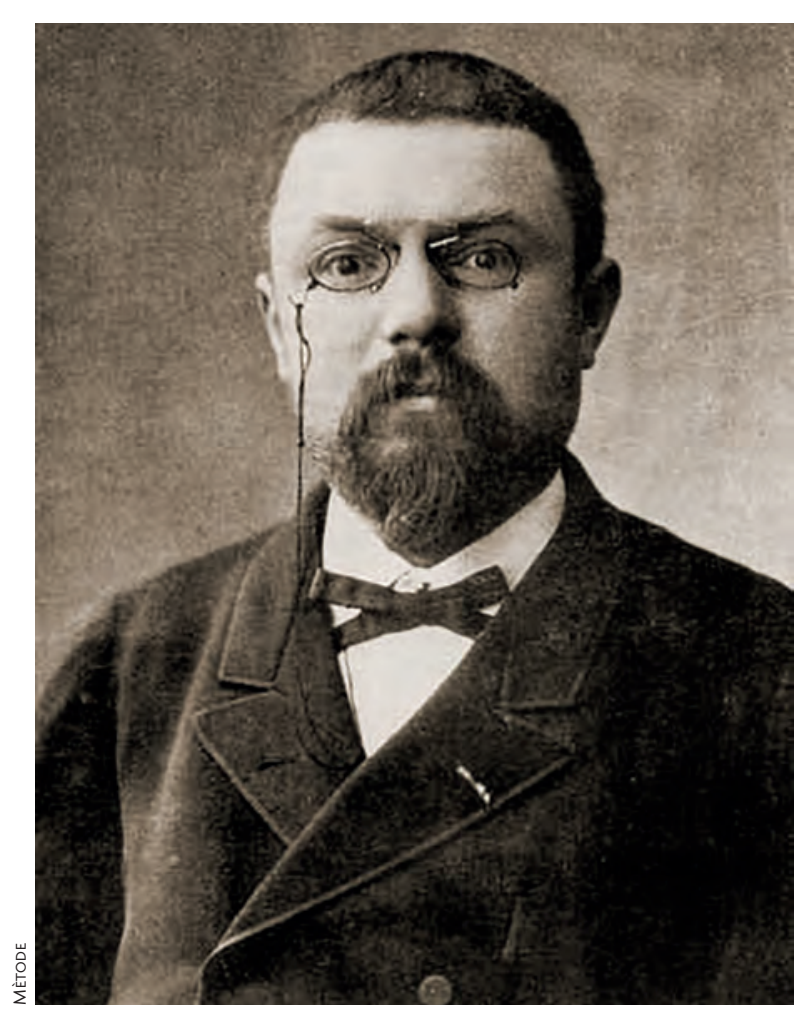

In 1904 the French mathematician Jules Henri Poincaré (1854-1912) proposed one of the most well-known problems in the history of mathematics, framed in one of its most abstract fields: topology.

them. For a topologist, a hollow sports ball is still a sphere, independently of how inflated it is. Its size does not matter either; it is a sphere whether it is used in rugby, tennis, table tennis, or football. But a ball is topologically different from a tyre or a metal nut, because these latter objects have a central essential hole. We could say that the artist Salvador Dalí acted as a topologist when he painted his melting clocks.

This branch of mathematics, widely known and used by theoretical physicists, was not fairly valued by general science until the recent discovery of topological insulators. These are new types of materials that insulate on the inside but are very good conductors on the outside. These properties are due to their shape, i.e. the topological structure which remains after deformations in the material. The theoretical physicists David Thouless, Duncan Haldane, and Michael Kosterlitz received the Nobel Prize in 2016 for their applications of topology to quantum physics in these new materials and this award brought this mathematical discipline to the foreground.

In order to understand the context of the conjecture, let us first analyse some basic concepts. A space is the place where physical phenomena occur, where points move following the laws of physics. Not all

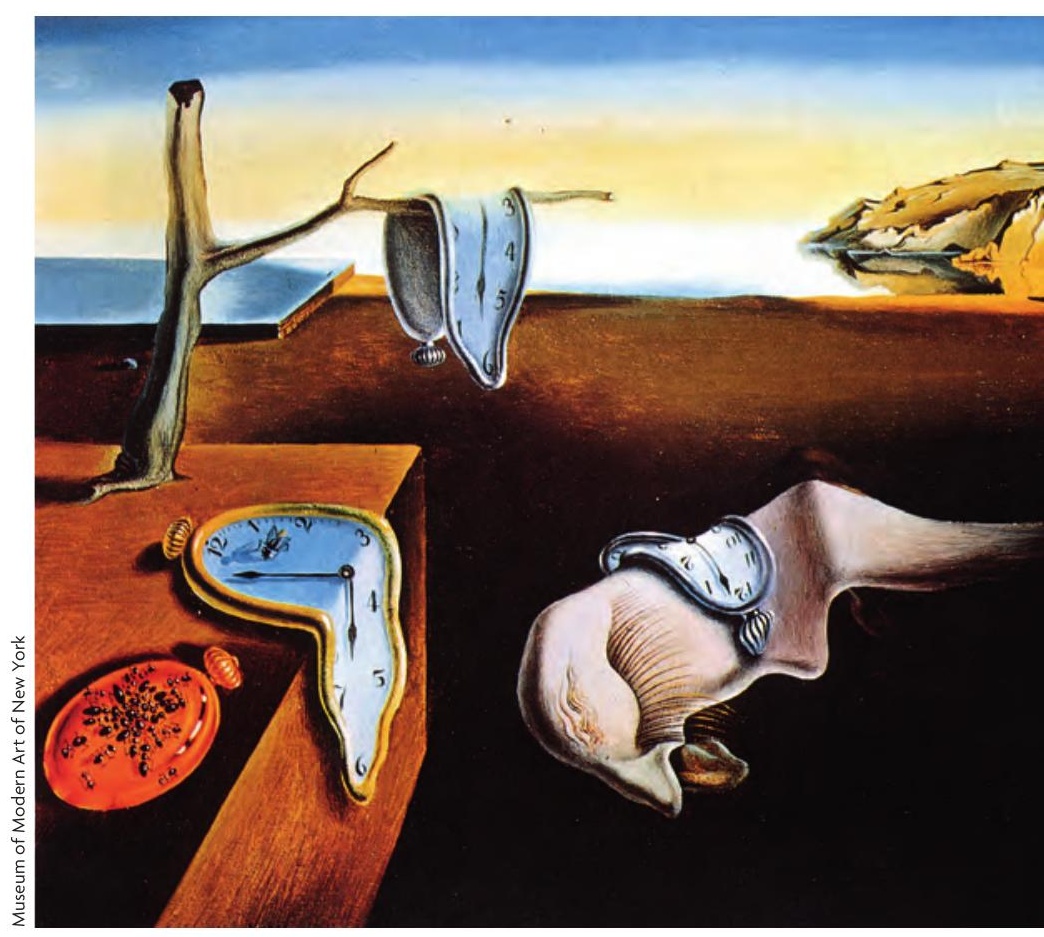

For a topologist, a hollow sports ball is still a sphere, independently of how inflated it is. Its size does not matter either; it is a sphere whether it is used in rugby, tennis, table tennis, or football. We could say that the artist Salvador Dalí acted as a topologist when he painted his melting clocks.

spaces allow for the same phenomena: dimension plays an important role. Intuitively, the dimension of a space is understood as the maximum number of independent directions or coordinates. Thus, a curve has dimension 1 , a surface has dimension 2 , and our environment has dimension 3 . We can imagine a variety of dimension 4 if we also consider time in a physical dimension 3 space.

Manifolds are the most interesting objects in topology. The mathematical concept of an $n$-dimension manifold (or $n$-manifold) is the abstraction of the $n$-dimensional space. Manifolds are spaces with similar characteristics at any point. That is to say, the local surroundings around a given point are analogous to the local environment around any other point, and analogous to the local situation of the classical physical $n$-dimensional space, i.e., each point is the centre of an $n$-dimensional ball.

Locally, a dimension 1 manifold is like an interval of a straight line, a dimension 2 manifold is like a disk, and a dimension 3 manifold is like a ball... If all the manifolds of the same dimension are topologically equivalent locally, what can we know about them globally? This question leads us to think about a global classification of manifolds. We would like to 
obtained can collapse at a point. The juxtaposition of $b$ and a loop such as $c$ provides a loop homotopic to $b$.

There is an important question we have not yet taken into account: a path can be followed in two directions. Then we say one is the inverse of the other. Note that if we juxtapose a path and its inverse, we get a loop that can be contracted to a point. In mathematical terms, we have obtained a group denoted as $\pi_{1}(T, P)$. A group is an algebraic structure consisting of a set and an operation between its elements that verifies three properties. Our set consists of the classes of closed paths and the operation is the juxtaposition. The class that can be contracted to a point is the neutral element or identity.
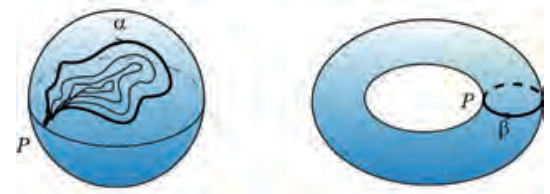

In topology, loops are closed paths that start and end in $P$. In the figure, we can observe examples of loops in the sphere (left) and the torus (right).

In general, the elements of the fundamental group $\pi_{1}(X, P)$ of a topological space $X$ based on a point $P$ of the space $X$ are the loop classes, closed paths starting and ending at point $P$, and their composition law is the juxtaposition. The identity is the class of paths $\alpha$ that can be continuously deformed contracting to point $P$, without ever leaving the manifold and preserving the ends at point $P$. A group is considered trivial if it is reduced to only the identity. It is easy to observe that every closed path $\alpha$ in the sphere represents the identity, because they can always be contracted to $P$. One can imagine that the path is an elastic band that shrinks during deformation. Therefore, the fundamental group of the sphere is trivial. But a path $\beta$ on another surface $F_{\mathrm{g}}$, different from the sphere that surrounds an essential hole, cannot be contracted to point $P$ without leaving the surface or breaking the path.

When the fundamental group of a manifold is trivial, the manifold is considered «simply connected». This is equivalent to saying that every closed path contracts continuously to one of its points. Thus, using this invariant, the fundamental group, we have characterised the two-dimensional sphere: the sphere $S^{2}$ is the only simply-connected closed surface.

Dimension 3. This topic interests, essentially, scientists because their curiosity leads them to crave knowledge about the global nature of world we inhabit, i.e. the shape of the universe. We live in a threedimensional space, so when we observe the manifold we inhabit globally, our situation is limited to local observation, as happened to Twodi, the inhabitant of the two-dimensional surface in the previous paragraph. We cannot visualise three-dimensional manifolds globally, so we must turn to the study of invariants in order to distinguish them. Manifold invariants are algebraic objects (numbers, polynomials, groups, etc.) that we associate with each manifold so that an invariant takes the same value in equivalent manifolds.

In the case of surfaces, the simplest one is the sphere $S^{2}$. If we look for a coordinate representation of the $n$-dimensional sphere, $S^{\mathrm{n}}$, we situate it in the $(n+1)$-dimensional Euclidean space, an ordinary space of $n+l$ dimensions with Euclidean metrics in which the shortest distance between two points is the straight line. In this Euclidean space, the sphere is the topological space whose points are at the same distance from a given point, for instance, the origin of coordinates.

$$
\begin{gathered}
S^{1}=\left\{\left(x_{1}, x_{2}\right) \in \mathbb{R}^{2} \mid x_{1}^{2}+x_{2}^{2}=1\right\} \\
S^{2}=\left\{\left(x_{1}, x_{2}, x_{3}\right) \in \mathbb{R}^{3} \mid x_{1}^{2}+x_{2}^{2}+x_{3}^{2}=1\right\} \\
S^{3}=\left\{\left(x_{1}, x_{2}, x_{3}, x_{4}\right) \in \mathbb{R}^{4} \mid x_{1}^{2}+x_{2}^{2}+x_{3}^{2}+x_{4}^{2}=1\right\} \\
S^{4}=\left\{\left(x_{1}, x_{2}, x_{3}, x_{4}, x_{5}\right) \in \mathbb{R}^{5} \mid x_{1}^{2}+x_{2}^{2}+x_{3}^{2}+x_{4}^{2}+x_{5}^{2}=1\right\}
\end{gathered}
$$

With this introduction, we can understand the Poincare conjecture: the sphere $S^{3}$ is the only simplyconnected closed manifold of dimension 3 .

It is easy to verify that the fundamental group of the three-dimensional sphere is, indeed, the trivial group. The problem is finding a three-dimensional manifold with a trivial fundamental group other than the sphere (that is, a counterexample to the Poincaré conjecture), or proving that any closed three-dimensional variety with a trivial fundamental group is topologically equivalent to the sphere (confirming the Poincaré conjecture).

This simple statement remained unverified and unrefuted for a century. But that time was far from wasted. The twentieth century was fruitful for its study of topological, geometric, and differential techniques applicable to 3-manifolds, which led to their profound understanding. As we did with surfaces, we will now consider only orientable 3-manifolds. Even though the initial framework of the Poincaré conjecture was exclusively topological, its solution involves Riemannian geometry and the differential equations of the Ricci flow.

In the twentieth century there were interesting discoveries regarding the Poincaré conjecture. Poincaré also defined the first homology group, the abelianization of the fundamental group; that is, the group obtained from the fundamental group after adding the commutativity of all its elements. Poincaré himself, in 

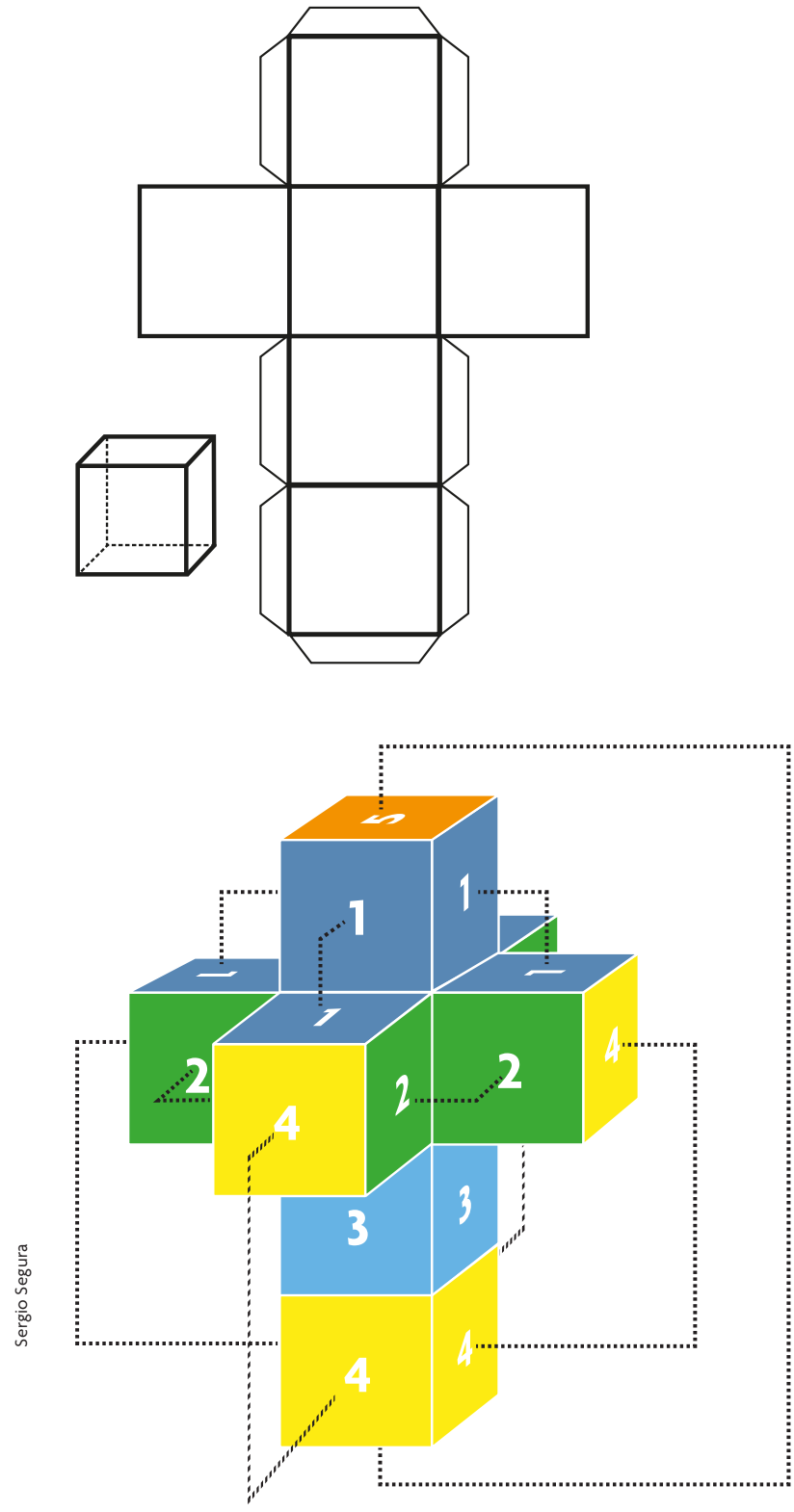

Imagining a three-dimensional object in a four-dimensional space is always difficult, but we can do it by analogy. Just as we can comprehend a three-dimensional object like a cube in a flat development, we can try to imagine a four-dimensional cube using its spatial development. In the top diagram, development of a cube in a plane; on the bottom one, development of a hypercube in a three-dimensional space (or rather, the projection of its spatial development on a plane). To obtain the figure, we need to join the faces with the same colour, which is impossible in our threedimensional space.

«IN THE 1980s, GEOMETRIC TECHNIQUES EMERGED THANKS TO THE MATHEMATICIAN WILLIAM THURSTON, FIELDS MEDAL 1982" his Cinquième complément à l'analysis situs (Poincaré, 1904), gave a negative answer to his first question of approximation in the Poincaré conjecture: is knowing that the first homology group of a 3-manifold is trivial sufficient to ensure that it is the 3 -sphere? That paper provides an interesting and beautiful counterexample to the issue: his famous dodecahedral manifold.

This manifold, known today as «Poincaré's homology sphere» or the «Poincaré dodecahedral space», can be defined as the set of regular dodecahedra (or, alternatively, regular icosahedra) inscribed into a two-dimensional sphere. It is the manifold resulting from identifying each face with its opposite in a solid dodecahedron, using a $\pi / 5$ twist. This manifold has a finite fundamental group of 120 elements, whose abelianization is the trivial group, and its universal cover is the sphere $S^{3}$. The dodecahedral manifold is obtained as the quotient of the sphere $S^{3}$ due to the action of a group of isometries with 120 elements. The point is to see the sphere as the boundary of a regular polytope of dimension 4 called the « 120 -cell». That is to say, the sphere $S^{3}$ tessellated with 120 regular spherical dodecahedra with $120^{\circ}$ dihedral angles, pasted together by their faces (720 pentagons), where each edge is shared between three dodecahedra. In total, there are 600 vertices and 1,200 edges. These dodecahedra are exchanged by a group of isometries. The quotient is Poincaré's homology sphere.

The Cinquième complément à l'analysis situs ends with the correct enunciation of the Poincaré conjecture, written as a statement. The text claims that what characterises the three-dimensional sphere is having a trivial fundamental group. The last sentence in the paper is: «Mais cette question nous entraînerait trop loin» ("But this question would take us too far").

Several mathematicians have confessed they have devoted part of their work to proving it or to finding a counterexample, although it has been said that every topologist has also tried to do so at some point. The need to find new arguments has allowed experts to find interesting procedures to construct all the closed 3 -manifolds, by analogy to known procedures for the construction of all closed orientable surfaces.

\section{NEW IDEAS: THURSTON}

Until 1980, the techniques used were either topological or combinatorial, but in the 1980s, geometric techniques emerged thanks to the mathematician William Thurston, Fields Medal 1982. The introduction of Riemannian geometry into a manifold consists in coherently defining a local metric in order to measure distances, angles, areas, etc. The intuitive idea is to 


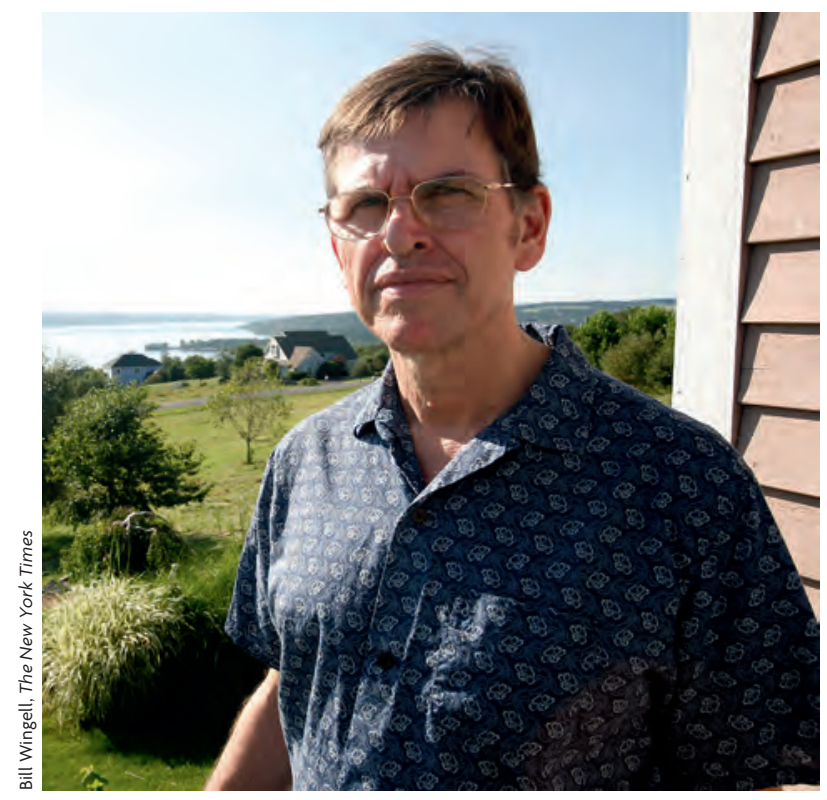

Until 1980, the techniques used were topological or combinatorial, but in the 1980s geometric techniques arrived thanks to the mathematician William Thurston.

harden the topological manifold to be able to use geometric techniques. If this is done on a surface, it can be done homogeneously so that the curvature is the same at each point on the surface. Thus, the two-dimensional sphere has a positive curvature; the torus (or $F_{1}$ ), has curvature 0 ; and surfaces $F_{\mathrm{g}}, g>1$, have a negative curvature. That is to say, the three Riemannian geometries - spherical (with a constant positive curvature), Euclidean (curvature zero), and hyperbolic (with a negative curvature) - are both necessary and sufficient to geometrise all closed surfaces.

To bring this to 3 -manifolds, we must divide the manifold into parts. The process is partly analogous to integer factorization. The idea is to divide the manifold in simpler pieces; these basic pieces are also referred to as «primes». The division consists in sectioning the manifold and attaching balls, thus obtaining simpler manifolds. For instance, in surfaces, the double torus can be divided first by a central circle, then adding two disks to cancel the created holes and two tori are obtained. The prime orientable surfaces are the sphere and the torus. Prime manifolds are those that cannot be divided further with this method. In dimension 3 , a manifold is prime if it is $S^{2} \times S^{1}$, or if any embedded sphere borders a ball.

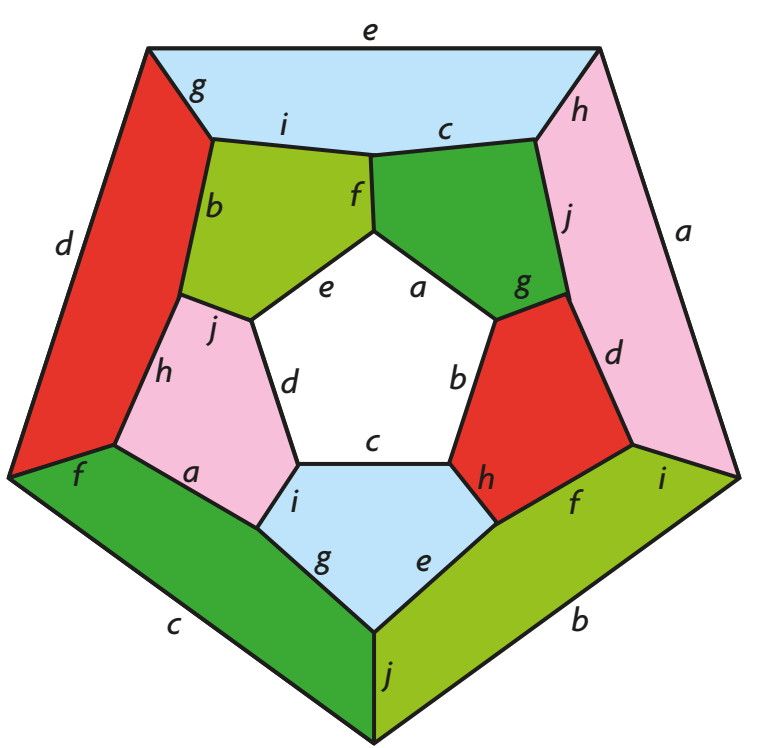

Poincaré's homology sphere is the result of identifying each face in a solid dodecahedron with the opposite one with $a \pi / 5$ twist, as in the figure, where the plane of projection is the boundary of the dodecahedron and opposing faces have the same colour.

Hellmuth Kneser proved that each closed 3-manifold different from $S^{3}$ contains a maximum finite number of spheres $S^{2}$ that divides the manifold into several pieces, so that if we attach a ball to each boundary sphere, prime manifolds are obtained (Kneser, 1929). Later, John Milnor proved that this decomposition into prime pieces is unique up to order (Milnor, 1962). Therefore, it seems reasonable to restrict the study to prime manifolds. A modification of Kneser's arguments permits cutting a prime manifold along a finite number of embedded incompressible tori to obtain simple pieces that do not contain more non-peripheral incompressible tori. A torus $\left(F_{1}\right)$ is «incompressible» if it cannot be simplified further, meaning that no simple essential closed curve in $F_{1}$ is continuously deformed in a point of the manifold. This collection of tori is also unique, as stated by the Jaco-Shalen-Johannson theorem

(Jaco \& Shalen, 1978; Johannson, 1979).

A manifold is «geometric» if it is the quotient of a geometry by a discrete group of isometries that act freely and discontinuously, and have a finite volume. Thurston proved that eight geometries were necessary to geometrise the interior of the simple pieces, and he conjectured that they were enough. More specifically, he established the conjecture of geometrisation in 
3-manifolds: each prime 3-manifold $\mathrm{M}$ is geometric or the interior of its simple pieces are geometric manifolds with only eight geometries.

Thurston's eight geometries - which are necessary and sufficient to geometrise 3-manifolds - perfectly described by Peter Scott (1983) and grouped here in three types, are:

\begin{tabular}{lll}
$\begin{array}{l}\text { Constant sectional } \\
\text { curvature }\end{array}$ & $\begin{array}{l}\text { Product } \\
\text { geometries }\end{array}$ & Twisted products \\
$\begin{array}{l}\text { Spherical } S^{3} \\
\text { (positive) }\end{array}$ & $S^{2} \times R$ & Nil \\
\hline $\begin{array}{l}\text { Euclidean } E^{3} \\
\text { (zero) }\end{array}$ & $H^{2} \times R$ & Sol \\
\hline
\end{tabular}

\begin{tabular}{ll}
$\begin{array}{l}\text { Hyperbolic } H^{3} \\
\text { (negative) }\end{array}$ & $\begin{array}{l}\text { The universal } \\
\text { cover of } S L(2 ; R)\end{array}$ \\
\hline
\end{tabular}

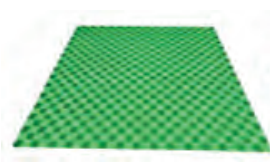

(a)

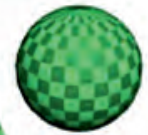

(b)

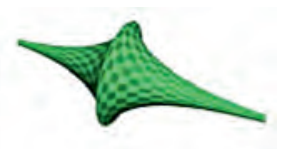

(c)
Figure illustrating the constant curvature surfaces in threedimensional space. They are the plane (a), with zero curvature; the sphere (b), with positive curvature; and the pseudosphere (c), which is the surface generated by a tractrix and has a negative curvature.

All the geometric manifolds with a geometry other than the hyperbolic have been classified. Therefore, after proving the geometrisation conjecture, we just need to classify hyperbolic manifolds.

The Poincaré conjecture is a special case in the geometrisation conjecture. Note that, of Thurston's eight geometries, only $S^{3}$, the one with constant positive sectional curvature, is compact. If a compact manifold is simply connected, it does not have an incompressible torus or essential spheres; therefore, the manifold is geometric and can only be $S^{3}$.

The decomposition in simple pieces has an inverse process, once simple pieces are given geometry. There are two ways to join two 3 -manifolds to obtain a new manifold. The first one, the «connected sum», consists in deleting a ball inside each of them and joining together the complements identifying the two resulting boundaring spheres. The second, used to join manifolds with boundary, is to identify two components of the boundary, one in each 3-manifold, by a homeomorphism. These unions are known as «wormholes» and connect geometric pieces. In the connected sum, we have a wormhole with spherical section; and in the second case, the wormhole's section is the surface used in the identification. In theory, a wormhole joining two space-time regions would allow us to travel in time and space. This idea has suggested stories, novels, and films where characters can move from one world to another crossing these (more or less invisible) frontiers that connect them.

Thurston proved his conjecture for a wide class of manifolds (Haken manifolds) that have enough complexity to be able to apply for his methods, which cannot be applied to simply-connected manifolds.

\section{THE RICCI FLOW: HAMILTON}

If we want to geometrise a 3-variety, we can start by describing a Riemannian metric in the manifold and making that metric change over time, trying to obtain a homogeneous metric for the whole manifold. If we consider surfaces, an easier dimension to understand these concepts, the idea is to think, for instance, in a topologically deformed sphere with its corresponding metric, and to turn it over time into a perfectly round sphere. The appropriate techniques for the development of these ideas require using differential equations, invented by Isaac Newton to explain how bodies move under the influence of an external force. In particular, the geometrist is interested in an analogous equation to the Fourier heat equation - the differential equation governing temperature changes - since a homogeneous distribution of temperature is reached over time. We want to use an equation to connect the change in geometry with a geometric quality such as curvature, in order to obtain a geometry with a homogeneous curvature distribution. Hamilton (1982) defined an equation for the Ricci flow including, on the one hand, the derivative of the metric tensor and, on the other, the Ricci tensor (related to curvature): $\partial_{t} g_{i j}=-2 R_{i j}$.

That is, the Ricci flow equation is analogous to the Fourier heat equation, but in a geometric context. Its goal is to homogenise curvature just as the heat equation homogenises temperature. If we start with a manifold with a crumpled metric geometry, we expect the flow to gradually correct the anomalies and reach a manifold with regular geometry.
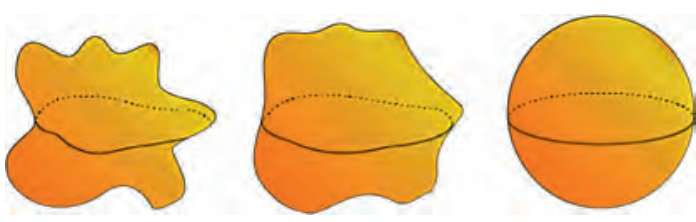

The Ricci flow equation is analogous to Fourier's heat equation, but in a geometric context. Its goal is to homogenise curvature just as the heat equation homogenises temperature. The idea is to think, for example, in a deformed topological sphere with its corresponding metric and, over time, transforming it into a perfectly round sphere. 
With these techniques, Hamilton proved powerful theorems, but he found obstacles to proving the Poincaré conjecture. He found that some singularities could be produced in the flow but was not able to solve them and he also had problems in analysing the situation when time goes to infinity in some cases. People interested in studying the Ricci flow can check the Lecture on the Ricci flow, published by Peter Topping in 2006 and available online'.

\section{THE SOLUTION: PERELMAN}

Perelman's announcement was surprising. He offered a positive solution to the Thurston geometrisation conjecture in his first two papers and a direct demonstration of the Poincaré conjecture in the third one (Perelman, 2002, 2003a, 2003b). His work is based on the Ricci flow and contains new ideas for solving problems related to singularities and in the step to the infinite limit. Understanding and verifying these results was the work of several groups of mathematicians who have published papers and books with detailed explanations of Perelman's work, including Bruce Kleiner and John Lott in Geometry and Topology, published in 2008. Although it was eventually published after other texts, it was the first to appear in arXiv, and to be freely consultable by the other teams. Other noteworthy work between 2006 and 2009 was that by John Morgan and Gang Tian, Huai-Dong Cao and Xi-Ping Zhu, and Laurent Bessières, Gérard Besson, Michel Boileau, Sylvain Maillot, and Joan Porti.

\section{THE POINCARÉ CONJECTURE IN HIGHER DIMENSIONS}

So far we have talked about $S^{1}, S^{2}$ y $S^{3}$, but nothing keeps us from broadening the dimension and considering $S^{4}, S^{5}, S^{n}$, and so on. In general, the $n$-sphere $S^{n}$ is defined as the set of vectors

$$
\left(x_{1}, x_{2}, \ldots, x_{n}, x_{n+1}\right) \in \mathbb{R}^{n+1}
$$

such that

$$
x_{1}^{2}+x_{2}^{2}+\cdots+x_{n}^{2}+x_{n+1}^{2}=1
$$

Therefore, in any dimension $n>1$ we can consider a problem analogous to the Poincaré conjecture, although without spatial intuition.

We cannot enunciate the Poincaré conjecture in dimension $n$ saying that the sphere $S^{n}$ is the only closed and simply-connected dimension $n$ manifold, because we know there are closed and simply-connected

\footnotetext{
${ }^{1}$ http://homepages.warwick.ac.uk/ maseq/topping_RF_mar06.pdf
}

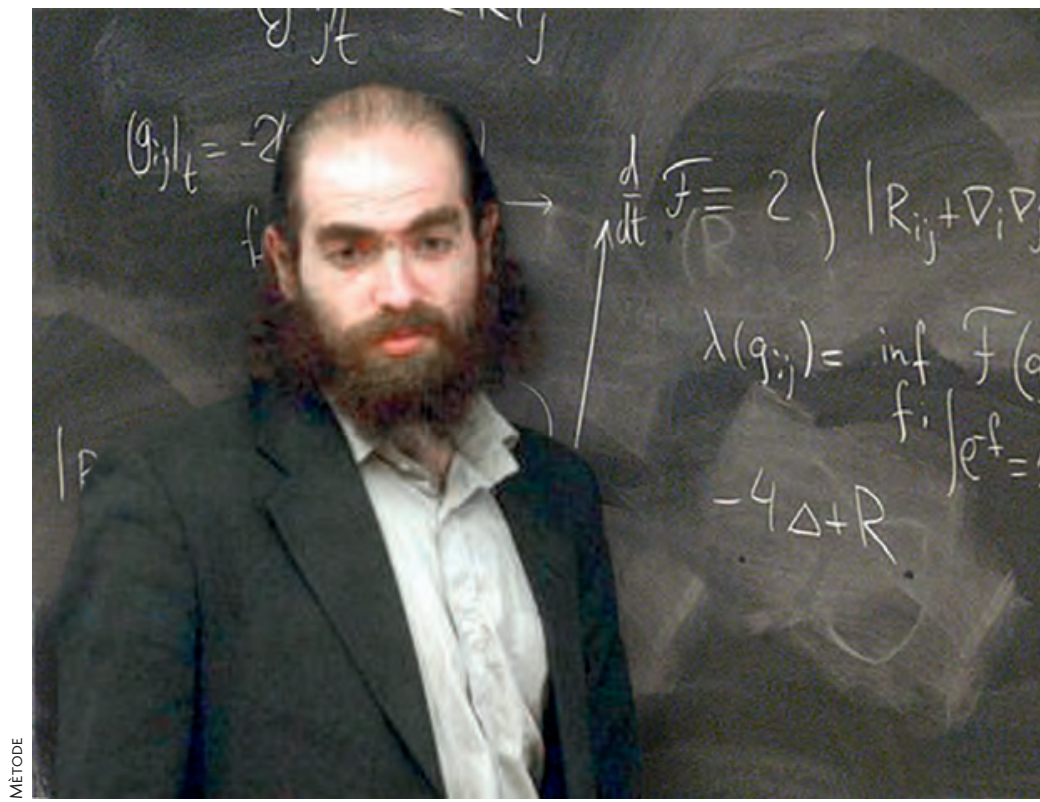

The Russian mathematician Grigori Perelman did not accept his Fields Medal in 2006 or the million dollar prize in 2010 for the solution of the Poincaré conjecture, one of the millennium problems.

$n$-manifolds $(n>3)$ that are not homeomorphic to the $n$-dimensional sphere (for instance, $S^{2} \times S^{n-2}$ ). Therefore, in dimension $n>3$ we have to generalise the fundamental group. A class of paths that start and end at point $P$ is a class of maps from $S^{1}$ to the $X$-manifold containing the point $P$ and it can be imagined as a circular rubber band in the manifold containing $P$. We have denoted it as $\pi_{1}(X ; P)$. With one more dimension, we can take spherical elastic membranes (like balloons) containing $P$ : they are classes of maps from $S^{2}$ to $X$, containing point $P$ and denoted as $\pi_{2}(X ; P)$. We could keep on increasing dimensions and define the homotopy group $\pi_{i}(X ; P)$ as the classes of maps from $S^{i}$ to $X$ containing point $P$.

Two spaces $X, Y$ are of the same homotopy type if all their respective homotopy groups are isomorphic: $\pi_{i}(X ; P) \cong \pi_{i}(Y ; Q)$. The formulation of the conjecture in dimension $n(\mathrm{CP} n)$ is: every closed $n$-manifold of the same homotopy type as that of the sphere $S^{n}$ is equivalent to the sphere $S^{n}$.

The conjecture was solved first for dimension $n>4$. Since 1960, several mathematicians have tried different versions of the Poincaré conjecture in dimension $n$ through different methods. In dimensions less than or equal to 3 it does not matter if we work with topological, combinatorial, or differentiable manifolds, but this is not the case with higher dimensions. The used manifold category and its corresponding methods is what distinguishes the different demonstrations. 


\section{REFUSAL AS A PROTEST}

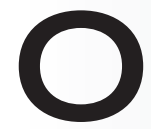
ne of the requirements for obtaining the Clay Institute prize is the publication of the problem in a specialised journal with a peer review process. But the Russian mathematician Grigori Perelman published his 2002 and 2003 works only on arXiv, the famous Cornell University website for scientific manuscripts. Those texts do not even mention the Poincaré conjecture, even though it would be a consequence of their results. When his papers were verified by the mathematical community and it was clear that they were correct, the International Mathematical Union awarded him a Fields Medal, which he should have received at the International Congress of Mathematicians held in Madrid in August 2006. But Perelman refused the prestigious award.

It is difficult to know why he rejected it. Perelman has always avoided the press, and few journalists have managed to meet and talk to him. Not even his biographer, Masha Gessen, was able to interview him about it. The most extensive and documented article about him might be the one published in The New Yorker in August 2006. 'The authors, Sylvia Nasar and David Gruber, explain the Poincaré conjecture, analyse different aspects of its solution (among them, an attempt to appropriate the solution), and describe an encounter with Perelman.

It seems that the keys to his refusal are fundamentally ethical. He said that the Fields Medal "was completely irrelevant to me. Everybody understood that if the proof is correct then no other recognition is needed.» But there is also a general reproach to the profession: Perelman stated that «there are many mathematicians who are more or less honest. But almost all of them are conformists. They are more or less honest, but they tolerate those who are not honest.» He added that uit is not people who break ethical standards who are regarded as aliens. It is people like me who are isolated.»

Perelman did not say at that time whether or not his objection to awards extended to the million dollar prize of the Clay Institute: «l'm not going to decide whether to accept the prize until it is offered.» However, when he was offered the prize in 2010 , he rejected it as well.

SERGIO SEGURA

\footnotetext{
1 www.newyorker.com/magazine/2006/08/28/manifold-destiny
}

The demonstration in dimension 4 was obtained twenty years later by Michael Freedman. In that same paper, he classified all the closed and simply connected 4-manifolds. Freedman also received a Fields Medal in 1986.

\section{LOOKING FOR THE SHAPE OF THE UNIVERSE}

Today we have some books that present the historical development of the topic with accessible content for university students. For example, The Poincaré conjecture: In search of the shape of the universe (O'Shea, 2007) explains the entire history of geometry, starting with the Pythagorean school in 500 BC, but taking into account Euclid, Gauss, Lobachevsky, and Bolyai's hyperbolic geometry, and the ideas by Riemann and Poincaré. It analyses twentieth century advances and explains Thurston's geometrisation conjecture. The subtitle of the book stimulates the reader's curiosity and promotes the study of threedimensional manifolds.

\section{REFERENCES}

Hamilton, R. (1982). Three-manifolds with positive Ricci curvature. Journal of Differential Geometry, 17(2), 255-306.

Jaco, W., \& Shalen, P. B. (1978). A new decomposition theorem for irreducible sufficiently-large 3-manifolds. In J. Milgram (Ed.), Algebraic and geometric topology (pp. 71-84). Providence: American Mathematical Society. doi: $10.1090 /$ pspum/032.2

Johannson, K. (1979). Homotopy equivalences of 3-manifolds with boundaries. Berlin: Springer-Verlag.

Kneser, H. (1929). Geschlossene Flächen in dreidimesnionalen Mannigfaltigkeiten. Jahresbericht der Deutschen MathematikerVereinigung, 38, 248-260.

Milnor, J. (1962). A unique decomposition theorem for 3-manifolds. American Journal of Mathematics, 84(1), 1-7.

O'Shea, D. (2007). The Poincaré conjecture: In search of the shape of the universe. New York: Walker Publishing Company.

Perelman, G. (2002). The entropy formula for the Ricci flow and its geometric applications. ArXiv. Retrieved from https://arxiv.org/abs/math/0211159

Perelman, G. (2003a). Finite extinction time for the solutions to the Ricci flow on certain three-manifolds. ArXiv. Retrieved from https://arxiv.org/abs/ math/0307245

Perelman, G. (2003b). Ricci flow with surgery on three-manifolds. ArXiv. Retrieved from https://arxiv.org/abs/math/0303109

Poincaré, H. (1904). Cinquième complément à l'analysis situs. Rendiconti del Circolo Matematico di Palermo, 18(1), 45-110.

Scott, P. (1983). The geometries of 3-manifolds. Bulletin of the London Mathematical Society, 15(5), 401-487.

\section{ACKNOWLEDGEMENTS}

Partial support came from MTM2013-45710-c2-1-p, MTM2016-76868-c22-p, and DGA/European Social Fund: Consolidated Group E15.

María Teresa Lozano Imízcoz. PhD in Mathematics from the University of Zaragoza (Spain) specialised in geometry and topology. Her research includes results on knot invariants, geometric structures in three-dimensional manifolds, universal links, and their related topics. She is an emeritus professor of the University of Zaragoza, tenured member of the Real Academia de Ciencias Exactas, Físicas, Químicas y Naturales of Zaragoza, and a corresponding member of the Royal Spanish Academy of Exact, Physical, and Natural Sciences. 